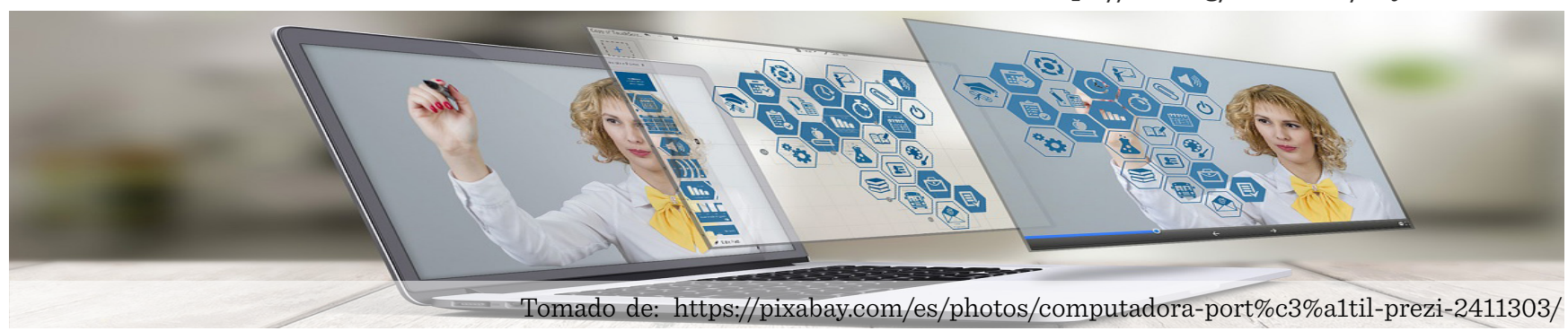

\title{
La formación de docentes en América Latina: entre la reproducción y la resistencia
}

\section{Teacher training in Latin America: between reproduction and resistance}

\author{
Rocío Jazmín Ávila-Sánchez, Ernesto Casas-Cárdenas
}

FECHA RECEPCIÓN: 11 oct 2021 FECHA DE ACEPTACIÓN: 8 de noviembre 2021

*Autor para correspondencia: ecasas@docentes.uat.edu.mx/Adscrpción:Universidad Autónoma de Tamaulipas

\section{Resumen}

En Latinoamérica, la formación de docentes es un tema nodal en la producción académica sobre educación. ¿Cómo se forman los que forman? Es una pregunta fundamental para entender los procesos y los alcances que tiene la educación en cada país. Y ello se refiere, no sólo a las prácticas pedagógicas, sino al contexto social en el que se forma la visión de los futuros docentes. Ante la interrogante de cómo se ha desarrollado el proceso de la formación de docentes, se plantea como objetivo principal de este artículo analizar el desarrollo de la Educación Normal en el contexto de los cambios políticos, sociales y económicos en América Latina, bajo la premisa de que a través de la implementación de cada uno de los modelos educativos se puede observar no sólo la perspectiva ideológica, sino el proyecto de nación que le subyace. Para desarrollar el tema se realiza un estudio desde la perspectiva comparada de países latinoamericanos, en los que con base en la literatura se observa la existencia de un segmento de la educación superior diferenciado que se enfoca en impartir carreras relacionadas con la educación, principalmente básica y en algunos casos media superior.

Palabras clave: Educación superior, modelos educativos, Escuelas Normales, apropiación social del conocimiento.

\begin{abstract}
In Latin America, the docent's formation is an important theme in the academic's production about education. ¿How they form those who form? It's a fundamental question to understand the process and the coverage that education has in each country. And this refers, not only to the teaching and pedagogical practices, but also to the social context in which it forms the vision of the teachers in the future. In view of the interrogant of how it has been developed the process of teacher's formation, the main objective or purpose of this article is to analyze the development of Normal school in the context of political, social and economic changes in Latin America, under the postulate that through the implementation of each one of the educational models it can be observe not only the ideological perspective but also the national project that underlies it. To develop the theme is carried out a study from the comparative perspective of Latin-American countries, they, based on literature they observe the existence of a segment of different higher education which focuses on teach careers related to education, mainly basic and in some cases middle higher education.
\end{abstract}

Keywords: Educational Law, Education system, Impact of European Educational System in subcontinent, Legislation.

Universidad Autónoma de Tamaulipas, Facultad de Derecho y Ciencias Sociales Victoria. 


\section{Introducción}

Ante los cambios sociales de mediados del siglo XIX en América Latina se planteó la necesidad de unificar los contenidos y las prácticas en la educación primaria, que para esos años aún eran sumamente diferenciadas, teniendo en la iglesia uno de los mayores proveedores de educación y en los varones el mayor número de asistentes. En países como Chile, se proclamaba que el fundamento de todas las ciencias es leer, escribir y contar, por lo que para lograr el crecimiento de un país era necesario que estos saberes estuvieran al alcance de todos y no solamente de las clases con mayores recursos económicos (Weinberg, 1984).

El proyecto para unificar contenidos requería necesariamente de la formación más o menos homogénea del profesorado que tendría a su cargo la labor de educar a los niños en sus primeros años escolares, asimismo al coincidir con el surgimiento de nuevas naciones, que en su mayoría vivían las primeras décadas de independencia, uno de los pilares del modelo educativo era consolidar el nacionalismo y enseñar conocimientos elementales, además de instruir en técnicas y oficios.

Y es que, en su origen las escuelas formadoras de docentes tenían la tarea de instruir en ciencias básicas y principios pedagógicos en el marco de un proyecto nacional, donde el magisterio estuviera identificado con las principales demandas sociales, adquiriendo además el compromiso con la formación de los futuros ciudadanos. En este sentido, es necesario resaltar que los modelos educativos están insertos en un ideario social, por lo que es necesario analizar el contexto histórico para comprender de una manera más amplia el significado del proceso educativo.

Bajo esta óptica es posible entender la educación impulsada por el estado a través de Escuelas Normales como una narrativa criolla para defender la idea del Estado nacional moderno. El asunto adquiere relevancia cuando se quiere comprender los modos en los que se puso en operación una biopolítica que permitiera ofrecerle un nuevo escenario al trabajo desarrollado por los educadores, cuyo encargo permanente fue ocuparse en producir inmateriales, tradiciones culturales, rituales, actos festivos y conmemoraciones (Álvarez \& Avendaño, 2015).

El origen del normalismo en América Latina El surgimiento de las primeras instituciones dedicadas a la formación del magisterio corresponde, como se apuntó, con los primeros años de vida independiente de los países latinoamericanos (Cuadro1) y a través de los nombres destinados a cada uno de los institutos se observa que en más de la mitad de los casos las escuelas estaban divididas con base en el sexo de los alumnos. Asimismo, destaca que en países como Honduras y Chile se presentan instituciones de capital privado desde los primeros años y en algunos casos la presencia de misiones extranjeras, como la alemana en Chile, Perú y Ecuador, la norteamericana en Argentina y la belga en Bolivia, en estos países se buscaba incorporar los modelos educativos implementados en otras latitudes, principalmente europeas, para mejorar el aprendizaje de los menores (Salgado, 2007).

En este marco, destaca la presencia del modelo lancasteriano de educación, cuyo principio de crear monitores capaces de replicar los conocimientos resultaba no sólo efectivo en las primeras etapas de la educación básica, sino también económico y accesible, ya que se enfocaba en la capacitación directa de aquellos que contaban con mayores conocimientos, quienes a su vez transmitían los conocimientos a los alumnos principiantes. Este modelo resultó preponderante en países como México, donde incluso la escuela lancasteriana se hizo cargo de la educación primaria durante más de dos décadas.

\section{Género y Normales}

En la implementación de las Escuelas Normales 
Cuadro 1. Primeras instituciones de Educación Normal en América Latina.

Table 1. First Normal Education Institutions in Latin America.

\begin{tabular}{|l|c|c|}
\hline País & Nominación & Año de Creación \\
\hline Perú & Escuela Normal de varones & 1822 \\
& Normal femenina & 1933 \\
\hline México & Escuela Normal & 1822 \\
\hline Costa Rica & Escuela de Formación de Maestros en Heredia & 1838 \\
\hline Chile & Escuela Normal de preceptores & 1842 \\
\hline Argentina & Escuela Normal de Paraná & 1870 \\
\hline Colombia & Escuela Normal Central de Bogotá & 1872 \\
\hline Panamá & Escuela Normal de Varones & 1872 \\
\hline Uruguay & Instituto Normal de Montevideo & 1885 \\
\hline Ecuador & Normal de varones & 1901 \\
\hline Honduras & Escuela Normal de señoritas & 1905 \\
\hline Nicaragua & Escuela Normal de varones & 1906 \\
\hline Bolivia & Escuela Normal de señoritas & 1908 \\
\hline Cuba & Instituto Pedagógico de Managua para varones & 1913 \\
\hline
\end{tabular}

Fuente: Elaboración propia con datos de: (Avalos, 2002; Inestroza 2003; Báez, 2004; Sequeira \& Robles, 2004; Sol, 2004; Salgado 2007, Figueroa 2016).

en países latinoamericanos fue común la división con base en género, ubicación geográfica o pertenencia étnica. De allí el nombre de normales de mujeres o varones, rurales y urbanas o indígenas como en el caso de la Normal de Warisata, fundada en Bolivia (Salgado, 2007). A esta especificidad se suma el papel que las Normales desempeñaron en la movilidad social, ya que para las familias de entornos rurales representaba una garantía laboral que sus hijos se incorporaran al magisterio, de la misma manera que lo significaba para las clases bajas del contexto urbano.

Aunque países como Perú, Ecuador y Panamá iniciaron la operación de Escuelas Normales exclusivas para hombres, la mayoría de los países optaron por crear escuelas para mujeres, con lo que se fue perfilando así una de las características más visibles del magisterio: la notable presencia femenina; en este sentido, como apunta Oresta López (2006) se trata de un fenómeno de discriminación social de la mujer que se traslada, se expande y se objetiva en las condiciones de un grupo socio profesional. Son incontables las obras que se refieren a la historia de la profesión magisterial sin reconocer como una característica importante su constitución mayoritariamente femenina (Arnaut, 1996; Meneses, 1998) y, pocas se han interesado por revisar las implicaciones que tiene una profesión feminizada en el complejo proceso que subyace al entramado actual del fenómeno magisterial 
La presencia predominante de mujeres como docentes en las primeras fases de la educación sugieren diversos puntos de análisis, por un lado, es necesaria la referencia a la teoría de la reproducción (Bourdieu \& Passeron, 2018) a través de la cual se puede inferir que la feminización del magisterio sería una consecuencia natural de la idea socialmente compartida respecto a la aptitud de las mujeres para el cuidado de los niños. Por otro lado, garantizaba también la posibilidad de acceder a un empleo, lo que a mediados del siglo XIX y durante la primera parte del siglo XX no era sencillo para la población femenina.

En México, durante el porfiriato y hasta la primera mitad del siglo XX, el discurso pedagógico de Pestalozzi que tenía gran influencia en la educación, planteaba como natural y conveniente el trabajo femenino para la educación de los niños, asimismo, ministros e interlectuales mexicanos, como Justo Sierra y Jesús Díaz Covarrubias, compartían estas ideas y es- pecialmente confiaban en que la copia del modelo estadunidense de feminización del magisterio también podría funcionar en México (López, 2006).

Sin embargo, se debe anotar qué durante esta época los salarios de las profesoras se encontraban por debajo del de sus homólogos varones, bajo el entendido, socialmente aceptado, de que los hombres requerían de un mayor ingreso para sufragar las necesidades de la familia a su cargo. En la actualidad, por ejemplo, y pese a que existe una igualdad de salarios establecida legalmente, se observa el incremento en el número de mujeres que se suman al magisterio en condiciones de semiprofesión, sobre todo en el estrato de educación inicial (que abarca de 0 a 4 años) y en el ámbito privado de educación básica, donde se perciben salarios muy por debajo de la media. Por ejemplo, en el caso de México, 93 de cada 100 profesores de preescolar son mujeres y 67 de cada 100 en el nivel primaria; sin embargo, si se analiza el porcentaje de mujeres que

Cuadro 1. Primeras instituciones de Educación Normal en América Latina.

Table 2. Percentage of female participation in the teaching profession by levels. (1980-1998).

\begin{tabular}{|l|c|c|c|c|}
\hline País & Preescolar & Primaria & Secundaria & Superior \\
\hline Perú & 99 & 60 & 46 & 22 \\
\hline México & 100 & 62 & 33 & N/D \\
\hline Costa Rica & 97 & 80 & 54 & 34 \\
\hline Chile & 89 & 73 & 51 & 22 \\
\hline Argentina & 99 & 91 & 67 & 35 \\
\hline Colombia & 96 & 76 & 44 & 24 \\
\hline Panamá & 100 & 63 & 54 & 34 \\
\hline Uruguay & N/D & N/D & N/D & 34 \\
\hline Ecuador & 95 & 65 & 42 & 18 \\
\hline Honduras & 100 & 74 & N/D & 29 \\
\hline Nicaragua & 99 & 85 & 57 & 36 \\
\hline Bolivia & 96 & 57 & 49 & N/D \\
\hline Cuba & 88 & 78 & 47 & 44 \\
\hline
\end{tabular}

Fuente: Elaboración propia con datos de: (Avalos, 2002; Inestroza 2003; Báez, 2004; Sequeira \& Robles, 2004; Sol, 2004; Salgado 2007, Figueroa 2016). 
ocupan la dirección de un plantel de educación primaria, la cifra desciende a 47\% (INEE, 2015).

Lo que indica una brecha de género que beneficia más a los hombres al momento de optar por un ascenso laboral. En el mismo sentido, la distribución de la participación de mujeres en los distintos segmentos educativos muestra un claro decremento conforme se avanza en el nivel de estudios; encabezan la lista Perú y Ecuador con una diferencia de 77 puntos porcentuales entre la presencia de mujeres en ejercicio de la docencia en el nivel de preescolar y en la educación superior, le siguen Colombia y Honduras con 72 y 71 puntos, respectivamente.

En el otro extremo se sitúa Cuba, aunque los 44 puntos que separan la participación de las mujeres no es un dato halagüeño. En el mismo sentido Chile tiene una diferencia de 67 puntos, seguido de Panamá con 66, Argentina de 64 puntos, Costa Rica y Nicaragua con 63 (Cuadro 2).

Esta diferencia también ha tenido un fuerte impacto en la investigación educativa, donde las Escuelas Normales poco aportan, compara tivamente hablando, con el resto de las Instituciones de Educación Superior, observándose una separación entre quién ejerce la docencia en educación básica y quien investiga acerca de ella, fenómeno que en los hechos ha implicado un alcance limitado de la producción durante las últimas décadas (Ruíz, Campos y Gamboa: 2019 y Siqueiros y Vera: 2020). Para ilustrar esta afirmación las cifras que sobre investigación educativa en México emite el Programa para el Desarrollo del Profesorado (Prodep) indican que solamente el $4 \%$ de los Cuerpos Académicos, (grupo de Profesores de Tiempo Completo que comparten objetivos y metas académicas, entre los que resalta la investigación) están adscritos a una Escuela Normal, el resto labora en Universidades e Institutos (Promep, 2021).

Aunque los datos con los que se cuenta pertenecen en algunos casos a casi dos décadas atrás, es posible observar que la tendencia en la feminización del magisterio ha sufrido pocos cam- bios, ya que las cifras oficiales proporcionadas por la Dirección General de Educación Superior para Profesionales de la Educación (Dgespe) señalan que en México 87,740 alumnos estudiaban en Escuelas Normales en el ciclo escolar 2017-2018, de los cuales poco más del 74 por ciento son mujeres.

\section{Las Escuelas Normales ante los cambios de modelo}

Las Normales desde su creación, han estado vinculadas directamente con el papel de reproducción de un orden determinado y tienen como uno de sus objetivos principales la consolidación de un patrón cultural homogéneo, en este sentido puede explicarse que hasta la fecha en algunos países de la región latinoamericana la formación de docentes esté encabezada por el Estado. Quizás por la importancia que tiene en los procesos de inculcación puede observarse, a partir de la literatura sobre el tema y la información estadística de las áreas encargadas de la educación en países latinoamericanos, que en los casos donde se mantiene la educación normalista existe un mayor control por parte del Estado sobre los planes, programas y por supuesto sobre el ejercicio presupuestal, en México, por citar un caso, se le considera un subsistema y comparativamente hablando tiene un reducido grado de autonomía frente al resto de las Instituciones de Educación Superior, hecho que de cierta manera ha incidido en la disminución de la matrícula durante las últimas décadas (Mendoza, 2018).

Por otra parte, los cambios en la configuración política y económica de la región, han tenido impacto significativo en el funcionamiento de las Escuelas Normales. Destaca el caso de las dictaduras militares, que en Chile implicaron el cierre de las Escuelas Normales y la transferencia de sus estudiantes y recursos a universidades cercanas; las cuales, cabe destacar, habían sido intervenidas por autoridades militares; así para principios de la década de 1980 las carreras de formación de profesores para todos los niveles fueron decretadas "no universitarias" y se ordenó su reestructuración en Academias Superiores o Institutos Profesionales. En Uruguay, 
durante el gobierno militar se crearon 21 Institutos de Formación Docente, a través de los cuales se buscaba debilitar la presencia del Instituto Pedagógico, desde donde se gestaban reclamos ante la llegada de los militares al poder.

En Bolivia, después del golpe de estado de 1971, encabezado por el ex ministro de Educación, General Hugo Banzer se decretó en 1975 la "Ley Normales" mediante la que se normó el sistema, modificando los planes y programas de estudios. Asimismo, resalta qué, en México durante la década de 1970, las Normales rurales fueron semillero de luchadores sociales que contaban con el apoyo de los habitantes de la región: Lucio Cabañas y Genaro Vázquez son ejemplos de maestros rurales que encabezaron movimientos de reivindicación de causas campesinas.

En efecto, las Escuelas Normales Rurales (ENR), creadas en México durante de década de 1920, representan un elemento de resistencia que requiere de un análisis con mayor profundidad; a manera de esbozo cabe mencionar que fueron implementadas con la encomienda de modernizar el campo a partir de transmitir conocimientos y educar a la población rural; si bien partían de las culturas locales, incluyendo el idioma, al modelo le subyace, desde entonces y hasta la fecha, la apuesta por un Estado unificador, prueba de ello es la existencia de libros de texto en idiomas originarios hasta cierto grado de educación primaria, con el objetivo de que los alumnos terminen insertándose en el modelo de cultura occidental.

Quizás por su relación con el campo y por las profundas raíces culturales de gran parte de los docentes, egresados y alumnos de las Normales Rurales, su existencia se ha convertido en uno de los bastiones críticos al modelo educativo neoliberal, a través del cual se pueden analizar los movimientos de resistencia (Civera, 2018). Y es que desde su fundación, los estudiantes de las normales rurales se agruparon en la Federación de Estudiantes Campesinos Socialistas de México (FECSM), destacando desde entonces por ser uno de los colectivos más politizados del magisterio. Su cercanía con los problemas del campo, la marginación, la explotación latifundista y el neocolonialismo aportó a los maestros rurales un sentido muy particular de solidaridad social (Flores, 2019).

En la década de 1960, el movimiento de estudiantes normalistas de Ayotzinapa, Guerrero, asociado a las figuras de Lucio Cabañas y Genaro Vázquez, estableció un parteaguas que vinculó los movimientos estudiantiles con la defensa de los derechos de los campesinos a través de guerrillas. Décadas más tarde los movimientos que buscan reivindicar el magisterio y la educación rural, pública y gratuita siguen estando vinculados con las ENR. La resistencia de los colectivos docentes egresados de estas escuelas, la constante denuncia a las prácticas colonizadoras y la defensa de las culturas originarias sigue siendo factor para el ataque sistemático desde el Estado a estos proyectos, siendo el caso más reciente el de Mactumactzá, Chiapas, en mayo del 2021.

El peligro que representa la organización colectiva vinculada a la tierra, tiene un referente concreto en las Normales Rurales, que originalmente formaban a sus estudiantes para enseñar a leer y a escribir, pero también como técnicos en agricultura para fortalecer el ámbito rural y mejorar la producción del agro mexicano. A casi un siglo de su fundación, lejos de ser vestigios de otra era, destaca su visión pragmática, una que en tiempos en que el campo languidece devastado tras décadas de abandono, haría bien en reexaminarse (Padilla, 2009).

Las políticas educativas que buscan la homogenización, en detrimento de las culturas originarias, para abonar a la construcción de un Estado occidental que se rige por valores impuestos desde los grupos hegemónicos que controlan el "mundo globalizado" se han materializado históricamente de formas diversas: la disminución presupuestal, la imposición de programas de estudio ajenos a las idiosincrasias latinoamericanas, el sesgo en el diseño de las evaluaciones que otorga ventajas a los habitantes de zonas urbanas y con mejores condiciones económicas. 
Así, de manera simultánea y con el ánimo de mejorar los índices de eficiencia impuestos por organismos internacionales para cuantificar el desempeño del sistema educativo se fueron desincorporando valores como la solidaridad, para privilegiar las competencias, la verticalidad en lugar de las redes horizontales que los docentes impulsaban en sus grupos para robustecer el concepto de colectividad y se priorizaron las materias tecnológicas e instrumentales en sustitución de aquellas con contenidos filosóficos que contribuían a crear generaciones cada vez más reflexivas (López, 2008).

Con la implementación de políticas de orden neoliberal, en América Latina las normales empezaron a ser absorbidas por universidades, o transformadas en institutos con características muy distintas a las que estructuraban el modelo normalista en décadas anteriores. En argentina la formación de docentes recayó en institutos superiores no universitarios que funcionan simultáneamente con los de nivel universitario; en Bolivia se han consolidado los institutos normales superiores que sustituyen a las antiguas escuelas normales, en Ecuador, las antiguas Escuelas Normales fueron transformadas en instituciones de educación superior no universitaria; en Chile, el normalismo llegó a su fin durante la dictadura militar de Augusto Pinochet.

En contraparte, en Uruguay la formación de maestros todavía se realiza en los Institutos Normales de Montevideo y en los de formación docente distribuidos en diferentes regiones del país, regidos por una autoridad específica y, finalmente en el caso de México se mantiene la estructura normalista con la vertiente por niveles, preescolar, primaria y secundaria, además de la modalidad por áreas, física, especial y artística; destacan la presencia de las normales rurales y las bilingües (especializadas en la formación de docentes hablantes de lenguas originarias), que se mantienen nominalmente, aunque con un presupuesto significativamente menor al que requieren.

\section{Conclusiones}

A través de este recorrido sobre realidades di- versas en Latinoamérica se pretende mostrar la importancia que la formación docente, bajo los principios de laicidad, gratuidad y obligatoriedad ha tenido en el desarrollo de la educación, sin embargo también se debe apuntar que ha sido a partir de la escuela desde donde se han gestado las marcadas desigualdades del sistema educativo nacional y latinoamericano, tema cuyo debate es imperativo y en el que es necesario analizar los proyectos de nación que subyacen a cada uno de los modelos educativos propuestos e implementados.

Durante las primeras décadas las normales representaron el campo ideal para la transmisión de conocimientos, pero también la promoción de la historia y el apego a la patria, valores que se constituyeron como ejes de las actividades escolares. Los docentes egresados de estas instituciones pasaron a formar parte de un sistema de colaboración social que se sustentaba en que a través de la educación sería posible consolidar un mejor país y una sociedad cohesionada en torno a valores cívicos.

Asimismo, los normalistas se convirtieron en defensores de la educación primaria gratuita, porque habían sido formados bajo la lógica de concebir a la educación como un bien público y un derecho universal. El cambio de modelo político y económico desdibujó los planteamientos sobre la gratuidad y el derecho a la educación y ésta se concibió también como una mercancía y un valor de cambio bajo los nuevos parámetros del mercado. Así, el papel rector que en un principio jugó el Estado en la educación pública, se espera que sea asumido por actores privados, una lógica diametralmente opuesta a los principios que dieron origen al actual sistema educativo, y que ahora coloca a la ganancia -traducida en eficacia y eficiencia- como el eje de la actividad educativa.

Dicho en otros términos y abarcando también a otras modalidades de educación superior en América Latina, la tendencia dominante ha instalado un concepto de calidad desde un paradigma tecnocrático y productivista, que reduce evaluación a control, confunde la calidad 
con ciertos indicadores cuantitativos y la evaluación con ciertos instrumentos de medida (Cano, Castro \& Sanguinetti, 2015).

Puede afirmarse que América Latina tiene la escolaridad que corresponde a sus grandes problemas culturales, sociales, económicos y políticos, pero sobre todo la brutal desigualdad (De Ibarrola, 2012). La óptica neoliberal, basada en la primacía del individuo por encima del colectivo, de lo cualitativo sobre lo cuantitativo y sobre la construcción de imaginarios ajenos a la identidad latinoamericana ha frenado el desarrollo de la región, abonando a la consolidación de modelos culturales propicios para los intereses de los grupos transnacionales que obtienen grandes ganancias económicas amparados bajo la premisa de traer modernidad y desarrollo; con la misma estructura discursiva que en la conquista.

Sin embargo, si se observa el trabajo de los docentes, existe un potencial enorme, basado en la riqueza colectiva que subyace al proceso de enseñanza-aprendizaje. La esencia de la educación normal en Latinoamérica está fundada en la solidaridad, la construcción colectiva de mejores condiciones de vida, el reconocimiento al entorno y el respeto al medio ambiente.

Asimismo, se pondera el objetivo emancipatorio de la educación: pensar, disentir y cuestionar; por ello resulta un ejercicio necesario volver la vista a los procesos educativos basados en el respeto a la identidad, la cultura y el sentido de pertenencia, de los cuales el modelo normalista ha sido un ejemplo en América Latina.

\section{Referencias}

Álvarez, L. y Avendaño, C. (2015) Historiografía de la Educación en Tamaulipas. Tomo I. México: Centro Regional de Formación Docente e Investigación Educativa.

Arnaut, A. (1996). Historia de una profesión. Los maestros de educación primaria en México 18871994. México: CIDE.

Avalos, B. (2002). Profesores para Chile: Historia de un Proyecto. Santiago: Ministerio de Educación.
Báez, M. (2004) Las Escuelas normales de Varones del Siglo XIX en Colombia, en Revista Historia de.la Educación Latinoamericana. Colombia Universidad Pedagógica y Tecnológica de Colombia, N. 6.

Bourdieu, P. y Passeron J. (2018) La reproducción, elementos para una teoría del sistema educativo. México: Siglo XXI editores.

Cano, A. Castro, D. \& Sanguinetti, M. (2015) Escenarios de la educación terciaria y superior en Uruguay: entre la diversificación integrada y la conformación de paisajes educativos duales. En Contrapunto. Educación: lo público, lo privado y lo común. Uruguay

Civera, A. (2018) La escuela como opción de vida. La formación de maestros normalistas rurales en México (1921-1945). Estado de México: El Colegio Mexiquense.

De Ibarrola, M., (2012). Los grandes problemas del sistema educativo mexicano. Perfiles educativos, 34 (spe), 16-28. México: Instituto de Investigaciones sobre la Universidad y la Educación México, UNAM.

Figueroa, C. (2016). Historia de la formación de educadores en América Latina. Centro investigaciones históricas y sociales Dr. Federico Brito Figueroa y Fundación Buria. Disponible en: https://www. academia.edu/33920559/Historia_de_la_Formaci\%C3\%B3n_de_Educadores_en_Am\%C3\%A9rica_ Latina_De_la_escuela_Normal_a_la_Universidad_ Pedag\%C3\%B3gica.

Flores, Y. (2019), "Escuelas Normales Rurales en México: movimiento estudiantil y guerrilla”, UAM Iztapalapa. Revista de Ciencias Sociales y Humanidades, núm. 87, año 40, julio-diciembre de 2019

INEE (2015). Los docentes en México. Informe 2015. Disponible en: https://www.inee.edu.mx/wp-content/uploads/2018/12/P1I240.pdf

Inestroza, J. (2003). La Escuela Hondureña en el Siglo XIX, Honduras: Fondo Editorial de la UPNFM.

López, F. (2008) Tendencias de la educación superior en el mundo y en América Latina y el Caribe. En Avaliação, 13, 267-291.

López, O. (2006) Las maestras en la historia de la Educación en México: contribuciones para hacerlas visibles. Sinéctica, Revista Electrónica de Educación. México: Instituto Tecnológico y de Estudios Superiores de Occidente.

Mendoza, J. (2018). Situación y retos de la cobertura del sistema educativo nacional. Perfiles Educativos, vol. XL, número especial, 11-52.

Meneses, A. (1998). Tendencias educativas oficia- 
les en México 1821-1911. México: CEE-UIA.

Padilla, T. (2009). Las normales rurales: historia y proyecto de nación. El Cotidiano. México: Universidad Autónoma Metropolitana- Azcapotzalco (154), 8593. Disponible en: https://www.redalyc.org/articulo. oa?id=32512736009

Promep. (2021). Cuerpos académicos Prodep. Disponible en: https://promep.sep.gob.mx/ca1/conceptos2.html

Ruíz, E., Campos, R. y Gamboa, A. (2019): Cuerpos Académicos en las Escuelas Normales: Perspectivas y obstáculos vigentes. Migración, cultura y estudios de género desde la perspectiva regional. Universidad Nacional Autónoma de México y Asociación Mexicana de Ciencias para el Desarrollo Regional A.C.

Salgado, R. (2007). La formación docente: de las normales a las universidades. En: Informe sobre la Educación Superior en América Latina y el Caribe 2000-2005. La metamorfosis de la educación superior. (171-182). Venezuela: Instituto Internacional de la UNESCO para la Educación Superior en América Latina y el Caribe (IESALC).

Sequeira, V. \& Robles, E. (2004). Las Primeras Escuelas Normales en el Perú. En: Revista Historia de la Educación Latinoamericana. Universidad Pedagógica y Tecnológica de Colombia, N.6.

Siqueiros, M y Vera, J. (2020) Caracterización de Cuerpos académicos de escuelas normales. Revista de Investigación educativa. Número 31 Julio-diciembre. México: Instituto de Investigaciones en Educación. Universidad Veracruzana.

Sol, R. (2004) Diagnóstico sobre la Formación de Docentes en Instituciones de Educación Superior en Costa Rica. Costa Rica: IESALC/UNESCO.

Weinberg, G. (1984) Modelos educativos en la historia de América Latina. Argentina: Kapelusz. 